\title{
Analysis of the treatment patterns and safety of early breast cancer patients during the COVID-19 pandemic
}

\author{
Feng $\mathrm{Li}^{1,2}$, Fengrui $\mathrm{Xu}^{1,2}$, Huiqiang Zhang ${ }^{1}$, Jianbin $\mathrm{Li}^{1,3}$, Tao Wang ${ }^{1}$, Shaohua Zhang ${ }^{1}$, Li Bian ${ }^{1}$, \\ Xiaopeng $\mathrm{Hao}^{1}$, Zefei Jiang ${ }^{1}$ \\ ${ }^{1}$ Department of Breast Oncology, The Fifth Medical Center of Chinese PLA General Hospital, Beijing, China; ${ }^{2}$ Department of Breast Oncology, \\ Academy of Military Medical Sciences, Beijing, China; ${ }^{3}$ Department of Medical Molecular Biology, Beijing Institute of Biotechnology, Academy of \\ Military Medical Sciences, Beijing, China \\ Contributions: (I) Conception and design: Z Jiang; (II) Administrative support: Z Jiang; (III) Provision of study materials or patients: All authors; (IV) \\ Collection and assembly of data: F Li; (V) Data analysis and interpretation: Z Jiang, F Li, F Xu; (VI) Manuscript writing: All authors; (VII) Final \\ approval of manuscript: All authors. \\ Correspondence to: Zefei Jiang. Department of Breast Oncology, The Fifth Medical Center of Chinese PLA General Hospital, No. 8 East Street, \\ Fengtai District, Beijing 100071, China. Email: jiangzefei@csco.org.cn.
}

\begin{abstract}
Background: An analysis of the impact of the coronavirus disease 2019 (COVID-19) on the patterns of treatment and the safety for patients with early breast cancer (EBC).

Methods: This was an observational real-world study. A total of 958 EBC patients who were admitted to two groups during the epidemic (Observation group: Q1 in 2020, n=422) and the similar time frame from last year (Control group: Q1 in 2019, $\mathrm{n}=536$ ). The primary end point of the study was the overall safety of treatments, including the proportion of neutropenia, fever, infection and infection of COVID-19. The secondary end point is the treatment patterns, referring to different treatment methods and the proportion of different regimens.

Results: Under the guidance of specific treatment strategies, the patient's treatment patterns have changed significantly. Among them, the proportion of albumin paclitaxel in the observation group was significantly increased $(55.8 \%$ vs. $13.0 \%, \mathrm{P}<0.001)$ compared with the control group, and the proportion of docetaxel was significantly reduced $(30.4 \%$ vs. $70.1 \%, \mathrm{P}<0.001)$. The proportion of anthracycline drugs and vinorelbine decreased (both $\mathrm{P}<0.05$ ). In the observation group, $22.5 \%$ of patients had neutropenia, which was significantly lower than the control group $(22.5 \%$ vs. $50.6 \%, \mathrm{P}<0.05)$, and the incidence of fever in the observation group was lower than that of the control group $(\mathrm{P}=0.05)$.

Conclusions: The patient's treatment patterns were significantly changed during COVID-19 pandemic, and optimized treatment strategy is suitable for breast cancer patients that can ensure the patient's treatment needs and overall safety.
\end{abstract}

Keywords: Coronavirus disease 2019 (COVID-19); breast cancer; treatment patterns; safety

Received: 28 May 2020. Accepted: 26 June 2020; Published: 30 July 2020.

doi: $10.21037 /$ tbcr-20-29

View this article at: http://dx.doi.org/10.21037/tbcr-20-29

\section{Introduction}

The coronavirus disease 2019 (COVID-19) caused an unprecedented alteration on the diagnosis and treatment of patients with breast cancer. Studies have shown that COVID-19 is more infectious for people with lower immunity $(1,2)$. Due to the influence of disease and treatment, the immunity of cancer patients is typically at a low level, especially for patients undergoing chemotherapy. For oncologists, it is not clear which treatment regimens are suitable and safe for breast cancer patients during the epidemic (3). 
We have formulated specific management measures and treatment strategies for breast cancer during the COVID-19 pandemic (4). In order to analyze the impact of COVID-19 on treatment patterns and the safety for patients with breast cancer under specific strategies, we compared the treatment and safety data for early breast cancer (EBC) patients who received treatment at the epidemic period (cohort 1: Q1 in 2020) and the same period last year (cohort 2: Q1 in 2019). The study protocol was conducted in accordance with the Helsinki Declaration of 1964 (revised 2008). We present the following article in accordance with the STROBE reporting checklist (available at http://dx.doi.org/10.21037/tbcr-20-29).

\section{Methods}

The study was conducted in accordance with the Declaration of Helsinki and the Harmonized Tripartite Guideline for Good Clinical Practice from the International Conference on Harmonization. This study was approved by the Institutional Review Board of the Fourth Hospital of Hebei Medical University. Informed consent was obtained from all individual participants included in the study.

\section{Study population}

This observational real-world study enrolled 958 patients with EBC from January 1, 2020 to March 31, 2020 and January 1, 2019 to March 31, 2019. All the patients were included in the outpatient or inpatient treatment at department of Breast Oncology, the fifth Medical Center of Chinese PLA General Hospital. The inclusion criteria for patients were as follows: the pathological diagnosis is invasive breast cancer, including complete pathological and immunohistochemical information. The patients included in the period from January 1, 2020 to March 31, 2020 were defined as the observation group, and the patients included in the period from January 1, 2019 to March 31, 2019 were defined as the control group.

\section{End points}

The primary end point of the study was the safety of treatment, including the proportion of neutropenia, fever, infection and infection of COVID-19. The secondary end point is the treatment patterns, including different treatment methods and the proportion of different chemotherapy regimens.

\section{Statistical analysis}

Non-normally distributed data is described by median, and categorical variables are described by percentage. Chisquare test, Fisher's exact probability method and Wilcoxon rank sum test are used to compare differences between groups. All data were completed using SPSS 19.0 (SPSS Inc., Chicago, IL, USA) for all statistical tests, which were two-sided tests with a significance level of 0.05 . When comparing the Chi-square test pairwise, the significance level was adjusted.

\section{Results}

\section{Clinical characteristics}

We analyzed the treatment differences for EBC patients who were treated in our center between the epidemic period (Observation group: Q1 in 2020, $\mathrm{n}=422$ ) and the same period last year (Control group: Q1 in 2019, $\mathrm{n}=536$, Table 1). Observation group patients had more hormone receptor (HR) positive patients $(62.6 \%$ vs. $54.5 \%, \mathrm{P}=0.01)$, and less triple negative breast cancer (TNBC) patients $(9.4 \%$ vs. $16.0 \%, \mathrm{P}=0.004)$. Other clinical characteristics such as disease stage and treatment stage did not show significant differences.

\section{Treatment patterns}

Forty-eight patients $(11.4 \%)$ in the observation group received neoadjuvant therapy, of which 2 patients $(0.5 \%)$ had completed neoadjuvant treatment, surgical treatment and received adjuvant therapy during the treatment period, 2 patients $(0.5 \%)$ have completed neoadjuvant and surgical treatment, and the adjuvant therapy has not started. Patients receiving neoadjuvant chemotherapy accounted for $10.7 \%(n=45)$, and neoadjuvant endocrine therapy accounted for $0.7 \%(\mathrm{n}=3) ; 374$ patients $(88.6 \%)$ received adjuvant therapy, of which 250 patients $(59.2 \%)$ received adjuvant endocrine therapy, 93 patients $(22.0 \%)$ received adjuvant chemotherapy, and $1.9 \%(\mathrm{n}=8)$ received only targeted therapy. In the control group, 58 patients (10.8\%) received neoadjuvant endocrine therapy, 55 patients (10.3\%) received neoadjuvant chemotherapy, and 3 patients $(0.5 \%)$ received neoadjuvant endocrine therapy. 478 patients $(89.2 \%)$ received adjuvant therapy. Among them, 298 patients $(55.6 \%)$ received adjuvant endocrine therapy, 99 patients $(18.5 \%)$ received adjuvant chemotherapy and 7 patients $(1.3 \%)$ received targeted therapy. 
Table 1 Characteristics of the patients at baseline

\begin{tabular}{|c|c|c|c|}
\hline Characteristic & Q1 in 2020 group $(n=422)$ & Q1 in 2019 group $(n=536)$ & $\mathrm{P}$ \\
\hline Median & 49 & 51 & \\
\hline Range & $30-88$ & $29-84$ & \\
\hline \multicolumn{4}{|l|}{ Molecular subtypes, n (\%) } \\
\hline HER2+ & $118(28.0)$ & $158(29.5)$ & 0.700 \\
\hline TNBC & $40(9.4)$ & $86(16.0)$ & 0.004 \\
\hline \multicolumn{4}{|l|}{ Stage, n (\%) } \\
\hline I & $102(24.2)$ & $129(24.1)$ & 1.000 \\
\hline \multicolumn{4}{|l|}{ Treatment stage, n (\%) } \\
\hline Only neoadjuvant therapy & $46(10.9)$ & $54(10.1)$ & 0.800 \\
\hline Neoadjuvant and adjuvant therapy & $2(0.5)$ & $4(0.7)$ & 0.700 \\
\hline Only adjuvant therapy & $374(88.6)$ & 478 (89.2) & 0.900 \\
\hline
\end{tabular}

\section{Proportion of chemotherapy drugs}

The observation group and the control group had significant differences in the choice of chemotherapy regimen (Figure 1). Compared with the control group, the proportion of albumin paclitaxel in the observation group was significantly increased $(55.8 \%$ vs. $13.0 \%, \mathrm{P}<0.001)$, and the proportion of docetaxel was significantly reduced (30.4\% vs. $70.1 \%, \mathrm{P}<0.001$ ). In addition, the proportion of other chemotherapeutic drugs such as anthracyclines and vinorelbine decreased (all $\mathrm{P}<0.05)$.

\section{Safety}

\section{Neutropenia}

Thirty-one of 138 patients (22.5\%) in the observation group experienced neutropenia, and the incidence was significantly lower than that in the control group $(50.6 \%, \mathrm{n}=78)$. Among them, the proportion of grade 3 to 4 neutropenia in the observation group and the control group were $5.8 \%(n=8)$ and $8.4 \%(\mathrm{n}=13)$, respectively (Table 2$)$.

\section{Fever}

A total of 4 patients in the 2020 Q1 cohort developed fever symptoms, which was significantly lower than the incidence of fever in the 2019 Q1 cohort (2.9\% vs. 9.1\%, $\mathrm{P}=0.05$, Table 2). Among them, 3 patients had transient fever, and the symptoms disappeared within 1 day after symptomatic treatment, which was considered to be drug fever. One patient had continued adjuvant chemotherapy for 6 cycles and developed fever symptoms on the 8th day after the 7 th cycle of continued treatment with albumin paclitaxel (200 mg, d1, d8) every 3 weeks, with a maximum body temperature of $38^{\circ} \mathrm{C}$, which lasted 3 hours and was accompanied by symptoms of bladder irritation. The nucleic acid test was negative for two consecutive times, which ruled out the possibility of COVID-19, and the final diagnosis was fever caused by urinary tract infection. The infection was controlled after giving antimicrobial treatment, and fever and urinary tract discomfort symptoms disappeared 2 days later.

\section{Discussion}

Ensuring the treatment needs and safety of breast cancer patients during the COVID-19 outbreak is a primary concern (5), but it's unclear which treatments are the safest proved. We compared the patterns of breast cancer patients treated during the COVID-19 period and the same period 


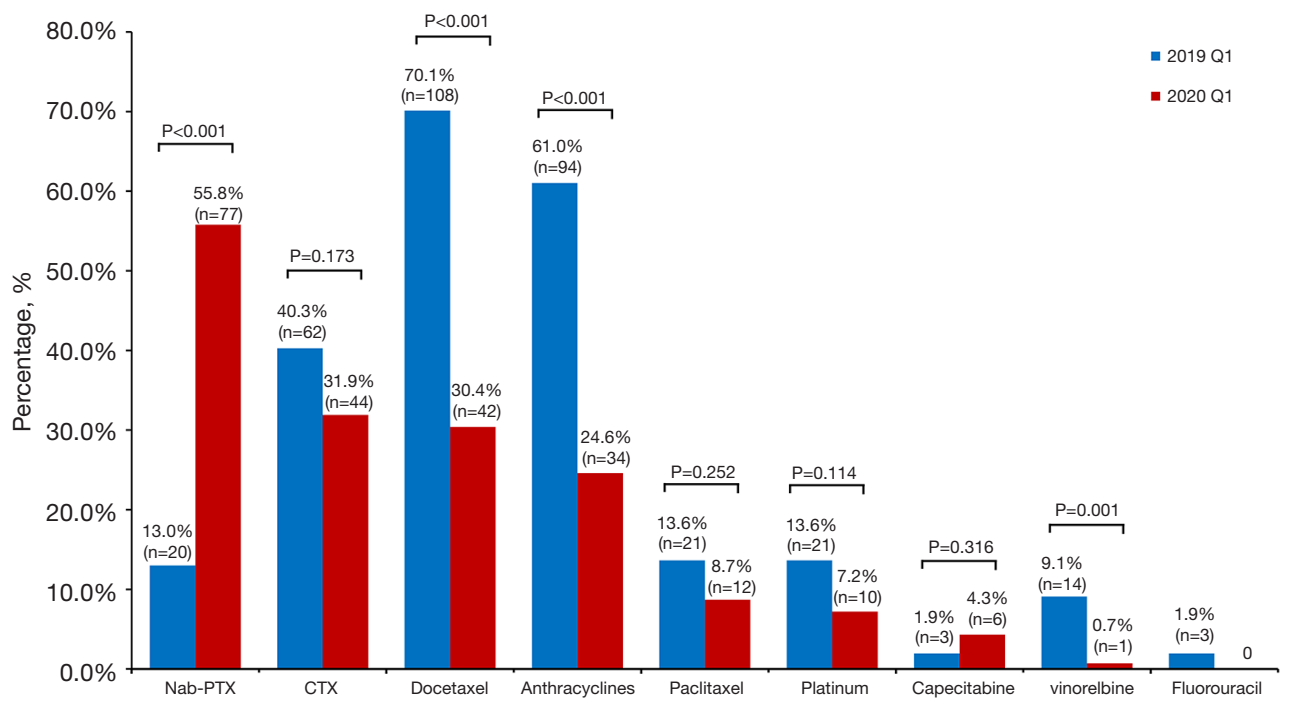

Figure 1 Difference for chemotherapy regimens between 2020 Q1 cohort and 2019 Q1 cohort. Nab-PTX, nanoparticle albumin-bound paclitaxel; CTX, cyclophosphamide.

Table 2 Adverse events of enrolled patients

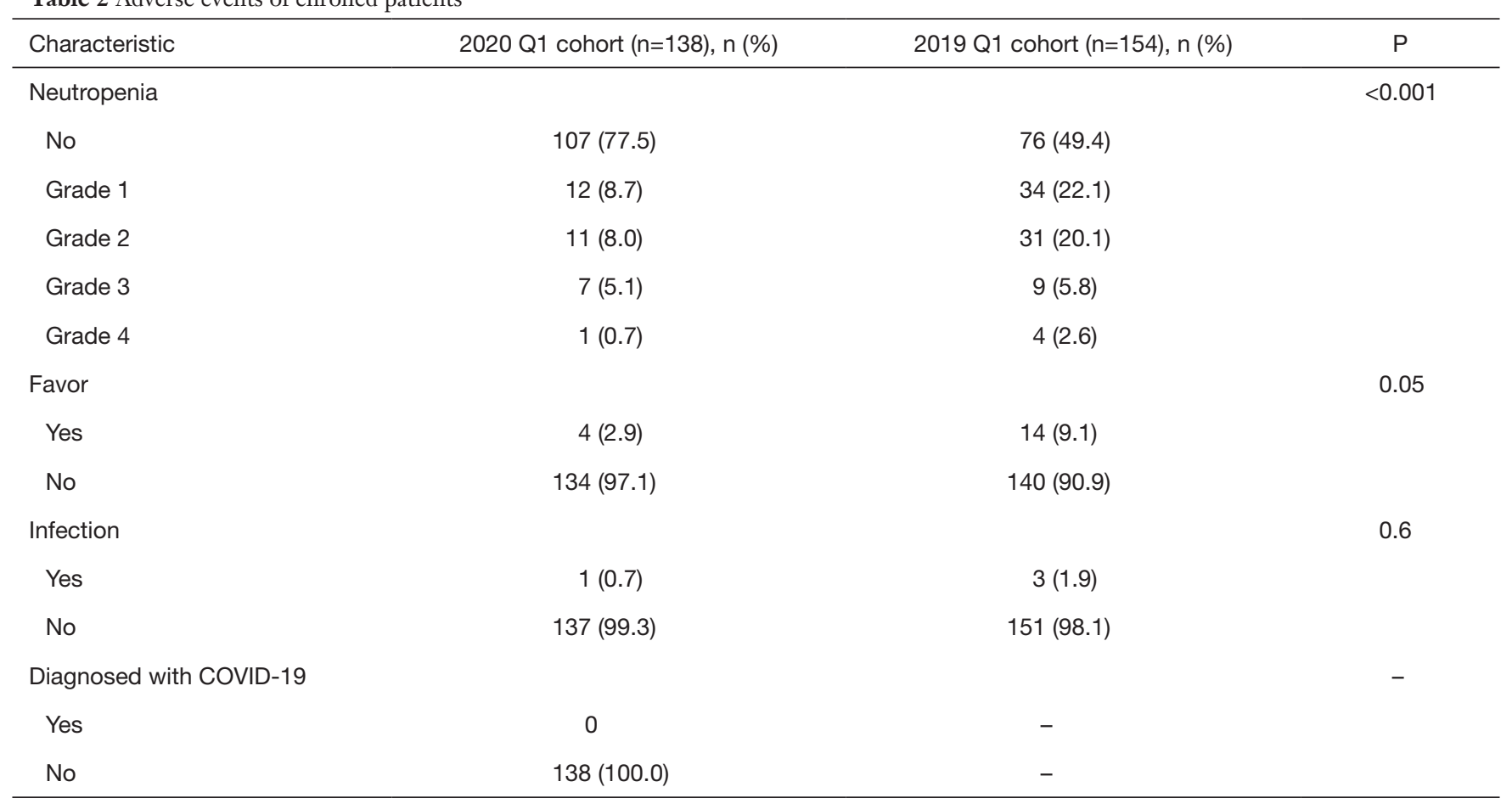

COVID-19, coronavirus disease 2019.

last year. The results showed that treatment patterns of EBC was significantly changed during the COVID-19 epidemic, and patients were treated safely under optimized treatment regimens such as mild chemotherapy.
The treatment options for breast cancer patients have changed significantly during the epidemic. In general, the application rate of low-risk bone marrow toxicity programs increased, while the application rate of medium-high 
risk programs decreased significantly. Taxus is the main chemotherapy drug for patients with $\mathrm{EBC}(6,7)$, different yews have different toxicity profiles $(8,9)$, both the CSCO guidelines (10) and NCCN guidelines (11) suggest that albumin paclitaxel has advantages over docetaxel in bone marrow toxicity, and we also witnessed the application rate of docetaxel decrease from $70 \%$ to $30 \%$, and the application rate of albumin paclitaxel increased from 13\% to $55 \%$, which made albumin paclitaxel become the highest proportion of drugs. The changes in treatment plan not only meets the patient's chemotherapy needs, but also avoids the added risk of causing patients to suffer from chemotherapy toxicity and infection.

The safety of treatment is a key factor influencing the treatment plan. Although we use treatment measures with lower toxicity, we are not sure the actual response of patients after treatment due to the prevalence of the special periods. At the same time, the safety requirements for treatment during the epidemic are higher than usual $(2,4)$. This study shows that no patient was diagnosed with COVID-19 during the epidemic, and the incidence of neutropenia or fever was significantly lower than the same period last year under the application of specific treatment strategies.

Neutropenia is the main adverse reaction of chemotherapy, which will affect the patient's immune system and increase the risk of infection. Under the protection of recombinant human granulocyte colony-stimulating factor (G-CSF), the incidence of grade 4 neutropenia in breast cancer chemotherapy patients is $2 \%$ to $10 \%$ (12). In this study, only one patient had grade 4 neutropenia during the epidemic, which was much lower than the incidence of agranulocytosis in the control group. We gave isolated protection to the patient with grade 4 granulocyte decline, and were injected with G-CSF, one day later, the patient's neutrophils gradually returned to normal, and no events such as infection or fever occurred.

Fever is an adverse reaction that requires special attention during the COVID-19 epidemic (13). During the epidemic, the appearance of fever symptoms will increase the psychological pressure of the patient, and further examination is required to differentiate the diagnosis (14). In this study, the incidence of fever during the epidemic was significantly lower than that of the control group. Of the 4 patients with fever symptoms, 3 patients were diagnosed with treatment-related drug fever and 1 patient was diagnosed with fever caused by urinary tract infection. Both rule out the fever caused by chemotherapy or COVID-19, which reduces the patient's psychological panic to a certain extent and the length of hospital stay.

In addition to safety considerations, the efficacy of treatment should not be ignored. At the same time, it is worth noting whether there is a phenomenon that efficacy is sacrificed because of excessive protection. Therefore, the long-term survival impact to the enrolled patients are under follow-up.

\section{Conclusions}

In conclusion, this study shows that with the support of specific strategies, it is safe for patients with EBC to receive the necessary anti-tumor treatment during the epidemic. Especially for those relatively low-risk treatment regimens, which can further improve short-term safety of patients and reduce the risk of infection. In the forthcoming stage, we need to continue to follow up the long-term efficacy of the patient to clarify the ultimate benefit of treatment decisions and provide an empirical reference for possible future public health emergencies.

\section{Acknowledgments}

Funding: None.

\section{Footnote}

Reporting Checklist: The authors have completed the STROBE Checklist. Available at http://dx.doi.org/10.21037/ tbcr-20-29

Data Sharing Statement: Available at http://dx.doi. org/10.21037/tbcr-20-29

Conflicts of Interest: All authors have completed the ICMJE uniform disclosure form (available at http://dx.doi. org/10.21037/tbcr-20-29). ZJ serves as an unpaid Editorin-Chief of Translational Breast Cancer Research. JL serves as an unpaid Managing Editor of Translational Breast Cancer Research. The other authors have no conflicts of interest to declare.

Etbical Statement: The authors are accountable for all aspects of the work in ensuring that questions related to the accuracy or integrity of any part of the work are appropriately investigated and resolved. The study was conducted in accordance with the Declaration of Helsinki 
and the Harmonized Tripartite Guideline for Good Clinical Practice from the International Conference on Harmonization. This study was approved by the Institutional Review Board of the Fourth Hospital of Hebei Medical University. Informed consent was obtained from all individual participants included in the study.

Open Access Statement: This is an Open Access article distributed in accordance with the Creative Commons Attribution-NonCommercial-NoDerivs 4.0 International License (CC BY-NC-ND 4.0), which permits the noncommercial replication and distribution of the article with the strict proviso that no changes or edits are made and the original work is properly cited (including links to both the formal publication through the relevant DOI and the license). See: https://creativecommons.org/licenses/by-nc-nd/4.0/.

\section{References}

1. Liang W, Guan W, Chen R, et al. Cancer patients in SARS-CoV-2 infection: a nationwide analysis in China. Lancet Oncol 2020;21:335-7.

2. Wang Z, Wang J, He J. Active and Effective Measures for the Care of Patients With Cancer During the COVID-19 Spread in China. JAMA Oncol 2020. [Epub ahead of print].

3. Yu J, Ouyang W, Chua MLK, et al. SARS-CoV-2 Transmission in Patients With Cancer at a Tertiary Care Hospital in Wuhan, China. JAMA Oncol 2020;6:1108-10.

4. Jiang ZF, Li JB. Ten hot issues of breast cancer during the COVID-19 pandemic. Zhonghua Yi Xue Za Zhi 2020;100:721-3.

5. van de Haar J, Hoes LR, Coles CE, et al. Caring for patients with cancer in the COVID-19 era. Nat Med 2020;26:665-71.

doi: $10.21037 /$ tbcr-20-29

Cite this article as: Li F, Xu F, Zhang H, Li J, Wang T, Zhang S, Bian L, Hao X, Jiang Z. Analysis of the treatment patterns and safety of early breast cancer patients during the COVID-19 pandemic. Transl Breast Cancer Res 2020;1:15.
6. Henderson IC, Berry DA, Demetri GD, et al. Improved outcomes from adding sequential Paclitaxel but not from escalating Doxorubicin dose in an adjuvant chemotherapy regimen for patients with node-positive primary breast cancer. J Clin Oncol 2003;21:976-83.

7. Mamounas EP, Bryant J, Lembersky B, et al. Paclitaxel after doxorubicin plus cyclophosphamide as adjuvant chemotherapy for node-positive breast cancer: results from NSABP B-28. J Clin Oncol 2005;23:3686-96.

8. Watanabe T, Kuranami M, Inoue K, et al. Comparison of an AC-taxane versus AC-free regimen and paclitaxel versus docetaxel in patients with lymph node-positive breast cancer: Final results of the National Surgical Adjuvant Study of Breast Cancer 02 trial, a randomized comparative phase 3 study. Cancer 2017;123:759-68.

9. Martín M, Seguí MA, Antón A, et al. Adjuvant docetaxel for high-risk, node-negative breast cancer. N Engl J Med 2010;363:2200-10.

10. Guidelines of Chinese society of clinical oncology breast cancer. Beijing: People's Medical Publishing House, 2019.

11. NCCN. Clinical practice guidelines in oncology (NCCN guidelines) 2020. V2, breast cancer. Available online: https://www.nccn.org/professionals/physician_gls/default. aspx\#breast

12. Xu F, Jiang Z. Prophylaxis of breast cancer chemotherapy for bone marrow toxicity: more individualized and safer. Ann Transl Med 2020;8:138.

13. Guan WJ, Ni ZY, Hu Y, et al. Clinical Characteristics of Coronavirus Disease 2019 in China. N Engl J Med 2020;382:1708-20.

14. Culakova E, Thota R, Poniewierski MS, et al. Patterns of chemotherapy-associated toxicity and supportive care in US oncology practice: a nationwide prospective cohort study. Cancer Med 2014;3:434-44. 